\title{
An exact DSatur-based algorithm for the Equitable Coloring Problem
}

\author{
Isabel Méndez-Díaz ${ }^{a, 1}$ Graciela Nasini b,1 Daniel Severín b,1 \\ a FCEyN, Universidad de Buenos Aires, Argentina, imendez@dc.uba.ar \\ b FCEIA, Universidad Nacional de Rosario y CONICET Argentina, \\ \{nasini, daniel\}@fceia.unr.edu.ar
}

\begin{abstract}
This paper describes an exact algorithm for the Equitable Coloring Problem, based on the well known DSATUR algorithm for the classic Coloring Problem with new pruning rules specifically derived from the equity constraint. Computational experiences show that our algorithm is competitive with those known in literature.
\end{abstract}

Keywords: equitable coloring, DSATUR, exact algorithm

\section{Introduction}

The Graph Coloring Problem (GCP) is a well known NP-Hard problem which has received many attention from the scientific community because of its large range of real applications and computational difficulty. Given a simple graph $G=(V, E)$ a $k$-coloring is a partition of $V$ into $k$ non-empty stable sets called color classes, denoted by $C_{1}, \ldots, C_{k}$, such that vertices in $C_{i}$ are colored with color $i$ for $i \in\{1, \ldots, k\}$. GCP consists of finding the chromatic number of $G$, denoted by $\chi(G)$, which is the minimum number $k$ of colors such that $G$ admits a $k$-coloring.

1 Partially supported by grants PIP-CONICET 241, PICT 2011-0817 and UBACYT 20020100100666 . 
There are many practical situations that can be modeled as a GCP with some additional restrictions. For instance, in scheduling problems, it would be desirable to assign a balanced workload among employees to avoid unfairness, or to assign balanced task load to prevent further wear on some machines more than others. The addition of this extra equity constraint gives rise to the Equitable Coloring Problem (ECP).

Formally, an equitable $k$-coloring (or just $k$-eqcol) of $G$ is a $k$-coloring satisfying the equity constraint, i.e. ||$C_{i}|-| C_{j}|| \leq 1$, for $i, j \in\{1, \ldots, k\}$ or, equivalently, $\lfloor n / k\rfloor \leq\left|C_{j}\right| \leq\lceil n / k\rceil$ for each $j \in\{1, \ldots, k\}$, where $n=|V|$. The equitable chromatic number of $G, \chi_{e q}(G)$, is the minimum $k$ for which $G$ admits a $k$-eqcol. The ECP consists of finding $\chi_{e q}(G)$ and it is also an $N P$-hard problem [4].

One of the most well known exact algorithms for GCP is Branch-andBound DSATUR, proposed by Brélaz in [2] and later improved by Sewell in [8]. This algorithm is still used by its simplicity, its efficiency in medium-sized graphs and the possibility of applying it at some stage in metaheuristics or in more complex exact algorithms like Branch-and-Cut. Recently, it was shown that a modification of DSATUR performs relatively well compared with many state-of-the-art Branch-and-Cut algorithms, showing superiority in random instances [7]. This fact makes nowadays research on DSatur-based solvers still important.

The goal of this work is to present a DSatur-based solver for the ECP. In the next sections, we review DSATUR algorithm and we propose new pruning rules specifically derived from the equity constraint. Finally, we report our computational experience.

\subsection{Notations and the DSATUR algorithm}

DSATUR is an implicit enumeration algorithm where each node of the tree corresponds to a partial coloration of the graph.

A partial $k$-coloring of $G, \Pi=\left(k, C_{1}, \ldots, C_{n}, U, F\right)$, is defined by a positive integer $k$, a family of disjoint stable sets $C_{1}, \ldots, C_{n}$ of $G$ such that $C_{j} \neq \varnothing$ if and only if $j \leq k$, a set of uncolored vertices $U=V \backslash\left(\cup_{j=1}^{k} C_{j}\right)$ and a list $F$ of their feasible color sets, i.e. for every $u \in U, F(u)=\{j \in\{1, \ldots, n\}$ : no vertex of $C_{j}$ is adjacent to $u$. Clearly, a partial $k$-coloring with $U=\varnothing$ is a $k$-coloring.

Given $\Pi=\left(k, C_{1}, \ldots, C_{n}, U, F\right), u \in U$ and $j \in\{1, \ldots, k+1\}$, we denote by $\langle u, j\rangle \hookrightarrow \Pi$ to the partial coloring of $G$ obtained by adding node $u$ to $C_{j}$, i.e. if $\langle u, j\rangle \hookrightarrow \Pi=\left(k^{\prime}, C_{1}^{\prime}, \ldots, C_{n}^{\prime}, U^{\prime}, F^{\prime}\right)$ then $k^{\prime}=\max \{j, k\}, C_{j}^{\prime}=C_{j} \cup\{u\}$, $C_{r}^{\prime}=C_{r}$ for all $r \neq j, U^{\prime}=U \backslash\{u\}, F^{\prime}(v)=F(v) \cap\{j\}$ for each $v \in U^{\prime}$ adjacent 
to $u$, and $F^{\prime}(v)=F(v)$ otherwise.

Given a maximal clique $Q=\left\{v_{1}, v_{2}, \ldots, v_{q}\right\}$ of $G$, we denote by $\Pi_{Q}$ the partial $q$-coloring defined by $C_{i}=\left\{v_{i}\right\}$ for all $1 \leq i \leq q$ and $U=V \backslash Q$.

DSATUR is based on a generic enumerative scheme proposed by Brown [3], outlined as follows:

INITIALIZATION: $G$ a graph, $\bar{c}$ an $U B$-coloring of $G$ and $Q$ a maximal clique of $G$.

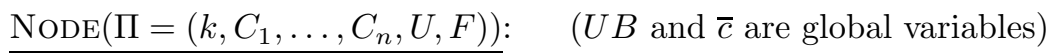

Step 1. If $U=\varnothing$, set $U B \leftarrow k, \bar{c} \leftarrow \Pi$ and return.

Step 2. Select a vertex $u \in U$.

Step 3. For each color $1 \leq j \leq \max \{k+1, U B-1\}$ such that $j \in F(u)$, do:

$\Pi^{\prime} \leftarrow(\langle u, j\rangle \hookrightarrow \Pi)$

If $F^{\prime}(v) \neq \varnothing$ for all $v \in U^{\prime}$, execute $\operatorname{Node}\left(\Pi^{\prime}\right)$.

It is not hard to see that the recursive execution of $\operatorname{NoDE}\left(\Pi_{Q}\right)$ finally gives the value of $\chi(G)$ into the variable $U B$ and an optimal coloring into $\bar{c}$ [2].

Based on this scheme, different algorithms for solving GCP have been evaluated by proposing different vertex selection strategies in Step 2. Two of them are due to Brélaz (DSATUR algorithm [2]) and Sewell (CELIM algorithm [8]). Recently, San Segundo proposed an improvement to Sewell rule (PASS algorithm [7]), which gave rise to a competitive solver with respect to many of the recent exact algorithms in the literature.

\section{EqDSatur: An exact algorithm for the ECP}

Let us notice that a trivial DSatur-based algorithm for the ECP can be obtained from the previous Brown's scheme, by changing the $U B$-coloring in the initialization by an $U B$-eqcol and adding the condition " $\Pi$ is an equitable coloring" in Step 1. However, using explicitly the equity property during generation of nodes we can avoid to explore tree regions that will not lead to an equitable coloring and therefore would be needlessly enumerated.

In the following lemma, we present necessary conditions for a partial coloring to be extended to an equitable coloring.

Lemma 2.1 Let $G$ be a graph of $n$ vertices and let $U B$ and $L B$ be, respectively, an upper and a lower bound of $\chi_{e q}(G)$. Let $\Pi$ be a partial $k$-coloring of $G$ such that $k<U B$ and $M=\max \left\{\left|C_{r}\right|: 1 \leq r \leq k\right\}$. Then, if $\Pi$ can be extended to an $r$-eqcol of $G$ with $k \leq r<U B$, the following properties hold: $(P .1)|U| \geq \sum_{r=1}^{k}\left(\max \left\{M-1,\left\lfloor\frac{n}{U B-1}\right\rfloor\right\}-\left|C_{r}\right|\right)^{+}$

(P.2) $M \leq\left\lceil\frac{n}{\max \{k, L B\}}\right\rceil$

In addition, property P.1 in the previous lemma also gives us a sufficient condition for a partial coloring with $U=\varnothing$ to be an equitable coloring: 
Lemma 2.2 If $\Pi$ is a partial $k$-coloring satisfying property $P .1$ and $U=\varnothing$ then $\Pi$ is a $k$-eqcol.

Our DSatur-based algorithm introduces the previous properties as modifications (written in boldface) into the Brown's scheme, in the following way:

INITIALIZATION: $G$ a graph, $\bar{c}$ an $U B$-eqcol of $G$, LB a lower bound of $\chi_{e q}(G)$ and $Q$ a maximal clique of $G$.

$\operatorname{Node}\left(\Pi=\left(k, C_{1}, \ldots, C_{n}, U, F\right)\right): \quad(U B$ and $\bar{c}$ are global variables $)$

Step 1. If $U=\varnothing$, set $U B \leftarrow k, \bar{c} \leftarrow \Pi$ and return.

Step 2. Select a vertex $u \in U$.

Step 3. For each color $1 \leq j \leq \max \{k+1, U B-1\}$ such that $j \in F(u)$, do:

$\Pi^{\prime} \leftarrow(\langle u, j\rangle \hookrightarrow \Pi)$

If $F^{\prime}(v) \neq \varnothing$ for all $v \in U^{\prime}$ and $\Pi^{\prime}$ satisfies P.1 and P.2, execute $\operatorname{Node}\left(\Pi^{\prime}\right)$.

We have the following:

Theorem 2.3 The recursive execution of $\operatorname{NoDE}\left(\Pi_{Q}\right)$ gives the value of $\chi_{e q}(G)$ into the variable $U B$ and an optimal equitable coloring into $\bar{c}$.

Proofs of the previous results are omitted due to lack of space.

In order to initialize our algorithm we use the heuristic NAIVE given in [4] for obtaining an initial $U B$-eqcol of $G$. The lower bound $L B$ is computed as in [6], i.e. as the maximum between the size of the maximal clique computed greedily and a relaxation of a bound given in [5].

Regarding the mentioned vertex selection strategies, we carried out benchmark tests over random instances and we concluded that PASS [7] is the best choice. We call EQDSATUR to our implementation using this strategy.

Another factor we take into account is in which order the nodes are evaluated in Step 3. All mentioned DSatur implementations for GCP evaluate first color $j=1$, then color $j=2$, and so on. We call EQDS E $_{1}$ to EDSATUR with this criterion. In addition, we consider another strategy based on sorting color classes according to their size in ascending order: if $\left|C_{i_{1}}\right| \leq\left|C_{i_{2}}\right| \leq \ldots \leq\left|C_{i_{k}}\right|$, we evaluate first $j=i_{1}$, then $j=i_{2}$, and so on. We call EQDS ${ }_{2}$ to EQDSATUR with this strategy.

\section{Computational experience}

Computational tests were carried out on an Intel i5 2.67Ghz over Linux O.S. Some details and tables were omitted due to lack of space. Instead, a summary of the most essential details are provided.

Our first experiment compares EQDS $\mathrm{E}_{1}$ against the "trivial" DSatur-based exact algorithm for the ECP mentioned at Section 2, in order to evaluate 
whether the time needed to check properties P.1 and P.2 compensate for the time wasted in exploring nodes of the enumeration tree where these properties do not hold. We noticed that $\mathrm{EQDS}_{1}$ really outperforms the trivial implementation. For instance, in medium-density random graphs of 70 vertices, EQDS is in average 25 times faster than the trivial implementation and is able to solve $21 \%$ more instances within 2 hours of execution.

The second experiment compares EQDS $\mathrm{E}_{1}$ and $\mathrm{EQDS}_{2}$ against "integer linear programming-based" solvers for ECP, a classical approach for developing exact algorithms. We consider the recent Branch-and-Cut proposed in [1] $\left(\mathrm{B} \& \mathrm{C}-L F_{2}\right)$ for which the authors report results on random instances up to 70 vertices in a $1.8 \mathrm{Ghz}$ AMD-Athlon platform. For random instances with 80 vertices, we compare EQDSATUR against CPLEX 12.1 solving the formulation for ECP given in [6] with the same initial bounds.

The following table reports the percentage of solved instances, average of relative gap and time elapsed for 100 random instances (10 per row, except results from [1]). An instance is considered not solved after the limit of two hours of execution. A bar "-" means no instance was solved. Each instance of $d \%$ of density is generated by considering a uniform probability $d$ that two vertices are adjacent to each other.

\begin{tabular}{|c|c|c|c|c|c|c|c|c|c|c|}
\hline \multirow{2}{*}{ Vertices } & \multirow{2}{*}{$\begin{array}{c}\text { \%Density } \\
\text { Graph }\end{array}$} & \multicolumn{3}{|c|}{$\%$ solved inst. } & \multicolumn{3}{|c|}{ \% Rel. Gap. } & \multicolumn{3}{|c|}{ Time } \\
\hline & & $L F_{2}$ & $\mathrm{EQDS}_{1}$ & $\mathrm{EQDS}_{2}$ & $L F_{2}$ & $\mathrm{EQDS}_{1}$ & $\mathrm{EQDS}_{2}$ & $L F_{2}$ & $\mathrm{EQDS}_{1}$ & $\mathrm{EQDS}_{2}$ \\
\hline 70 & 10 & 100 & 100 & 100 & 0 & 0 & 0 & 109 & 0 & 0 \\
\hline 70 & 30 & 0 & 100 & 100 & 18 & 0 & 0 & - & 0 & 0 \\
\hline 70 & 50 & 0 & 100 & 100 & 8,2 & 0 & 0 & - & 16,2 & 16,1 \\
\hline 70 & 70 & 100 & 100 & 100 & 0 & 0 & 0 & 273 & 31,2 & 32,1 \\
\hline \multirow[t]{2}{*}{70} & 90 & 100 & 100 & 100 & 0 & 0 & 0 & 11 & 0 & 0 \\
\hline & & CPLEX & $\mathrm{EQDS}_{1}$ & $\mathrm{EQDS}_{2}$ & CPLEX & $\mathrm{EQDS}_{1}$ & $\mathrm{EQDS}_{2}$ & CPLEX & $\mathrm{EQDS}_{1}$ & $\mathrm{EQDS}_{2}$ \\
\hline 80 & 10 & 100 & 100 & 100 & 0 & 0 & 0 & 5,7 & 0 & 0 \\
\hline 80 & 30 & 0 & 100 & 100 & 20 & 0 & 0 & - & 23,7 & 17,6 \\
\hline 80 & 50 & 0 & 100 & 100 & 24 & 0 & 0 & - & 477 & 424 \\
\hline 80 & 70 & 10 & 70 & 70 & 16 & 10 & 10 & 5769 & 1715 & 1653 \\
\hline 80 & 90 & 100 & 100 & 100 & 0 & 0 & 0 & 690 & 12,5 & 12,3 \\
\hline
\end{tabular}

Our algorithm is able to solve more instances than CPLEX and, without considering the difference of platforms, B\&C- $L F_{2}$. Also, EQDS $\mathrm{S}_{2}$ seems to be a little better than $\mathrm{EQDS}_{1}$ in terms of time.

The last experiment compares EQDS $\mathrm{E}_{1}$ and $\mathrm{EQDS}_{2}$ against CPLEX and $\mathrm{B} \& \mathrm{C}-L F_{2}$ on 24 benchmark instances reported in [1]. 16 instances have been solved by CPLEX, EQDS ${ }_{1}$ and EQDS 2 in less than two seconds. B\&C- $L F_{2}$ solved these 16 instances with an average of 62 seconds and never outperforms the other three algorithms. Instance queen8_8 have been solved by CPLEX in 654 sec., by B\&C- $L F_{2}$ in 441 sec., by EQDS ${ }_{1}$ in 7.5 sec. and by EQDS ${ }_{2}$ in 
1.1 sec. Instances miles1000 and miles750 have not been solved by EQDS ${ }_{1}$, but have been solved by B\&C- $L F_{2}$ in 267 and 171 sec. respectively, and by CPLEX and $\mathrm{EQDS}_{2}$ in less than a second.

On the other hand, neither $\mathrm{EQDS}_{1}$ nor $\mathrm{EQDS}_{2}$ could solve 5 instances. In particular, 3 of these instances (3-FullIns_3, 4-FullIns_3, 5-FullIns_3) are hard to solve by enumerative schemes, as reported in [7], so in our opinion, EQDSATUR presents the expected behaviour.

We want to remark that EQDSATUR has also been able to solve DIMACS benchmark instances which are not mentioned in [1]. For instance, queen9_9 has been solved by $\mathrm{EQDS}_{1}$ and $\mathrm{EQDS}_{2}$ in less than 10 minutes. In contrast, CPLEX could not solve it in the term of 4 hours of execution. Another example is myciel5 which has been solved in less than a sec. by $\mathrm{EQDS}_{1}$ and $\mathrm{EQDS}_{2}$ but CPLEX needed 149 sec. to solve it.

From these results, we conclude that $\mathrm{EQDS}_{2}$ is highly competitive with respect to the algorithms available in the literature for the ECP.

\section{References}

[1] Bahiense L., Y. Frota, T. F. Noronha and C. Ribeiro. A branch-andcut algorithm for the equitable coloring problem using a formulation by representatives, Discrete Appl. Math., In Press.

[2] Brélaz, D. New methods to color the vertices of a graph, Comm. of the ACM 22 (1979) 251-256.

[3] Brown, J. R. Chromatic scheduling and the chromatic number problem, Manag. Sci. (Part I) 19 (1972) 456-463.

[4] Furmańczyk H. and M. Kubale. The complexity of equitable vertex coloring of graphs, J. Appl. Comp. Sci. 13 (2005) 95-107.

[5] Lih K-W and B-L Chen. Equitable coloring of trees, J. Combin. Theory, Series B 61 (1994), 83-87.

[6] Méndez-Díaz I., G. Nasini and D. Severin. A polyhedral approach for the Equitable Coloring Problem. Discrete Appl. Math., In Press.

[7] San Segundo, P. A new DSATUR-based algorithm for exact vertex coloring, Comput. Oper. Res. 39 (2012) 1724-1733.

[8] Sewell, E. C. An improved algorithm for exact graph coloring, DIMACS Series in Discrete Mathematics and Theoretical Computer Science, AMS, Providence, Rhode Island 26 (1996), 359-373. 